\title{
The Extracorporeal Bio-Xeno Perfusion (myelo-timo-spleen) in Multi-organ Supportive Therapy (MOST) as a Modulator of Energy, for Immuno Correction Compensatory Anti-Inflammatory Response Syndromes, and Persistent Inflammation, Immuno suppression, Catabolism and Multi-organ Dysfunction
}

Vasiliev $\mathrm{I}^{{ }_{1}}$, Vasilieva $\mathrm{M}^{1}$, Vasilieva $\mathrm{I}^{1}$, Ilia $\mathrm{C}^{2}$, Tatiana $\mathrm{G}^{2}$ and Viorel $\mathrm{N}^{2}$

${ }^{1}$ Private Health Care Institution "Via- Intosana", Republic of Moldova

2State University of Medicine and Pharmacy "Nicolae Testemitanu" of the Republic of Moldova

*Corresponding author: Vasiliev I, Private Health Care Institution “Via- Intosana”, Republic of Moldova, E-mail: ilievasiliev@gmail.com

Citation: Vasiliev I, Vasilieva M, Vasilieva I, Ilia C, Tatiana G, et al. (2015) The Extracorporeal Bio-Xeno Perfusion (myelo-timo-spleen) in Multi-organ Supportive Therapy (MOST) as a Modulator of Energy, for Immuno Correction Compensatory Anti-Inflammatory Response Syndromes, and Persistent Inflammation, Immuno suppression, Catabolism, and Multi-organ Dysfunction. J Anesth Pati Care 1(1): 104. doi: 10.15744/2456-5490.1.104

Received Date: July 23, 2015 Accepted Date: November 12, 2015 Published Date: November 13, 2015

\begin{abstract}
The use of bio-perfusion as active method of energo-modulation and suspension of PICS expansion, which depends on the elimination of hypo(an)ergic mitochondria performed through a lysosomal clearance (mitophagy) so as to maintain the eu-ergic mitochondria which are able to contribute to the formation of cell energy, and ultimately, to the whole body as macroergic system. In this regard, since 1984, in order to remove this problem and restore mitochondrial energy and in order to treat patients suffering from CARS, PICS, MODS, or croniosepticemy/MSOF, as they were called back then, extracorporeal bio-perfusion has been performed. Its aims were: 1) To purify plasma and intracellular glicocalicsul biomembranes of toxic endogenous (STE) and microbial decay products; 2) The immuno-modulation of immune dissonance; 3) Immuno-detoxification; 4) The energy modulation - using extracorporeal xenograft donor organs, and namely the thymus, the bone marrow and the spleen (central and peripheral organs of the immune system). In the research we have taken into account the following facts: 1) CARS, PICS and MODS are unsolved problems which put limitations in the evolution of treatment and which are characterized by a high mortality and increased financial costs; 2) Performing the many organs supportive therapy- (MOST), in order to reduce mortality, has led to diametrically opposite results, generating an increase in the energy consumption and causing endogenous energy-deficit; 3) The absence within medical literature of MOST prosthetic models of central nervous system, the immune and energy system functions. Scientists from Florida, USA, described a new syndrome the persistent inflammation, immune suppression and catabolism syndrome (PICS). For the treatment we offer extracorporeal perfusion through bio xenogeneic (the central organs of the immune system: thymus and bone marrow, and peripheral: spleen) donor organs pig. Which provides mechanical, biological detoxification, improvement of humoral and cellular immunity, reduce inflammation and catabolism, and fulfill energy modulation.

KeyWords: The compensatory anti-inflammatory response syndrome; The persistent inflammation; Immune suppression and catabolism syndrome; The multiple organ dysfunction syndrome; The systemic inflammatory response syndrome; Mixed antagonistic response syndrome; Local inflammatory response syndrome; Mitophagy; Many organs supportive therapy; Extracorporeal bio-xeno perfusion (myelo-timo-spleen) perfusion; Energo-modulator; Xenocitokino (leikino) transfusion
\end{abstract}

\section{Introduction}

The variety of therapeutic strategies of immune are prolonged and/or chronic paralysis generated by the mitochondria energy deficit, which require large financial cost and has a high mortality rate, constitutes one of the new fundamental study issues in modern medicine. There is attested a heightened interest for the following syndromes: the compensatory anti-inflammatory response syndrome (CARS), the persistent inflammation, immune suppression and catabolism syndrome (PICS) and the multiple organ dysfunction syndromes (MODS). Scientists involved in the therapeutic strategy of this problem do not have a common view in the approach to the "receptor-target" treatment. Élie Metchnikoff launched the hypothesis according to which somatic diseases and malignant tumors are of contagious origin, that diabetes is caused by parasites of malignant tumors and microbial pathogens. In 1947, he wrote: “... in the course of time, we will probably discover not only the parasites of common diseases, such as the infectious ones, but also the parasites of totally different kind of diseases.... Modern researches have shown that up to $80 \%$ of all oncopathology cases are associated with infectious agents. The purpose of medicine to eradicate the infectious diseases (plague, cholera, typhoid and typhus, smallpox and natural polio) has not disappeared. Instead, at the end of the twentieth century 
ukemia, the hantavirus pulmonary syndrome, campylo-bacteriosis, X-infections, etc.). In its turn, the antibiotic therapy has resulted in a high resistance to antibiotics and caused a new problem - the nosocomial infections. The developing of infectious MODS led to a high mortality rate throughout human history. By 1970, the infectious agents were deemed exclusive factors that contributed to the development of MODS. Later, in 1980-1990, the infectious origin of MODS became less important, and by late 1980s W. Shoemaker, et al. replaced it with an alternative hypothesis, the oxygen-dependent occurrence of MODS, and in 1990s, the occurrence of MODS was attributed to the systemic inflammatory response syndrome (SIRS) introduced by R.C. Bone [1], which, as demonstrated, causes severe and non-infective MODS [2], including: burns, trauma, pancreatic necrosis, onco-toxicity, hypoxia and ischemias, including all states of shock. The further studies showed that homeostatic balance between SIRS and CARS is balanced through a confrontational mixed antagonistic response syndrome (MARS) that has the function to prevent the outbreak of MODS. These processes are performed also by the immune system, which in situations of immune incongruence, causes endotoxemia and immuno-endotoxicosis. The MODS, regardless of their origin, being accompanied by immune imbalances, mitochondrial energy resources and medical technologies, generate acute or prolonged/chronic MODS, a situation that has led scientists from Florida to detect and describe a new syndrome- the persistent inflammation, immune suppression and catabolism syndrome (PICS). Within the mechanisms against anti-harmful alteration there is an observed an antagonism of maintaining balance between local inflammatory response syndrome (LIRS), SIRS and CARS balanced by MARS. In the case of prevalence of SIRS, and thus the pro-inflammatory mediators (IL-1, 6, 8, TNF, IFN- $\gamma$ ) a patient develops a "storm" of cytokines leading to the danger of clinical manifestations such as hyper-ergic Schwartzman-Sanarelli reaction, Waterhouse-Friderichsen syndrome or in the case of massive destruction of microbes, the evolution of the Jarisch-Herxheimer therapeutic shock, that leads to acute MODS. In the case of prevalence of CARS, and namely of anti-inflammatory mediators (IL 4,10,13, etc.), the patient develops an immune paralysis, where the chronic infection causes a persistent hypo-ergic inflammation syndrome. F. Moore and his colleagues named it "the persistent inflammation, immune suppression, and catabolism syndrome" - PICS, that is similar to the clinical evolution of AIDS and develops a chronic MODS. We will examine the hypo(an)ergic immune-paralytic uncompensated CARS model able to generate PICS. In its presence, the slow infections, even the saprophytic or opportunistic flora, become aggressive and invade the body with endo and exogenous microbial toxins that destroy the host, causing chronic MODS due to a lack of resistance energy observed in immune suppression, catabolism, extreme myelopoiesis and impaired autophagy (mitophagy). Modern medicine provides sufficient models and several classifications of treatment using immune preparations in cases of immunodeficiency. A.S. Simbirtsev, et al. have researched the therapy with cytokines in view of modulating the immune system (GM-CSF, IL-2) in the appropriate infection and AIDS; IL-1, IL-2 viral hepatitis, herpes, bacterial infections (tuberculosis, purulent sinusitis, abscess, phlegmon, pancreatitis, osteomyelitis, etc.), the IFN- $\alpha$ viral hepatitis, human papillomavirus, and the like; IFN- $\gamma$ in granulomatous disease, tuberculosis, chlamydia, viral infections; IFN- $\beta$ in infectious asthma-allergic. In order to reduce the expression of HLADR receptors, W.D. Docke, et al. (1997) studied the (IFN- $\alpha$ ) cytokines and the stimulating factors of (GM-CSF) colonies. In 2010, E. Said, et al. applied antibodies against the interleukin IL-10 [3]. Many research papers reflect the relationship between the HLA-DR receptors, the IL-10 receptors, the immune paralysis and HIV infection. Also in 2010, S. Inoue, et al. described the anti-apoptotic effect of IL-15 in the reverse of dysfunctional monocytes. In 2004, D. Brealey, et al. identified that restoring normal mitochondrial function by removing the damaged mitophagy presents one of the key components of successful treatment of PICS. In 2010, Spruijt N. E., et al. defined, via the evidence-based medicine, only three pharmaceuticals (immunoglobulins, IFN- $\gamma$, glucan), which significantly reduce infection and mortality. In the case of the PICS syndrome there is attested a sudden decrease in the level of $\operatorname{IgG}$, which increases by means of exogenous administration of IgG, which, in its turn, increases the IgG-dependent antigen presentation. The absence in the case of PICS of the lymphocyte antigen presentation by inducing the expression of HLA-DR, restores the IFN- $\gamma$. Glucan, as a component of the inner wall of the Saccharomycces cerevisiae bacteria, contributes to the reduction of immune suppression by reducing the elimination of prostaglandins by macrophages and by activating the proliferation of bone marrow. However, the successful treatment of CARS/PICS is very modest and it has been proven that trying to restore the immune system function in hypo(an)ergic and extended/chronic MODS has had almost no results at all. Likewise, similar tests meant to treat early MODS and spontaneously generated SIRS, were not successful.

\section{Materials and Methods}

Since 1984, there were analyzed the following categories of hypo(an)ergic patients: 1) Patients that were weak from the very beginning, in which it was registered during their hospitalization a decreased appetite and weight loss, the "Weight loss", the "body mass index-BMI" and the "creatinine-growth" index-ICG), hipo-albumin- and hipoprealbuminemia; 2) Non-reactive patients characterized by low resistance which were frequently affected by any trivial infections (hypothermia, leukopenia and lymphopenia); 3) Patients with persistent inflammations (ranging from 14 days in hospital and continuing for a long term accompanied by a spread of loco-regional outbreaks and their generalization) in whose biological fluids there has been detected growth of bacteria strains that were confirmed by bacteriological tests and in which markers of sepsis have been reported [4]. Also, there has been estimated the number of bacterial cells, the endogenous intoxication, inflammation, the immunosuppression and the catabolism [5]. In order to correlate the research issues with the up-to-date information through the prism of modern visions, some of our information obtained in 1984 was conformed with the PICS clinical determinants described by L.F. Gentille, et al. (2012) [6], and namely: a prolonged period (hospitalization in an intensive care unit for a period exceeding 14 days); inflammation (CRP $>150 \mathrm{mg} / \mathrm{dl}$ ); immune suppression (total lymphocyte count $<800 / \mathrm{mm}$ ); catabolism (loss of weight (Weight loss) $>10 \%$ of base- 
there appeared some infections that are dangerous (legionellosis, ehrlichiosis, HIV-infection, yersiniosis, viral fever, Marburg, Lassa, Ebola, Lyme disease, enterotoxigenic escherichioses, a number of viral intestinal diseases, hepatitis E, C, D, F and G, the T cell lebaseline during the hospitalization and body mass index $(\mathrm{BMI})<18$, index "increased creatinine" (ICG) $<80 \%$, albumin $<3 \mathrm{~g} /$ dl, pre-albumin, $20 \mathrm{mkg} / \mathrm{dl}$ ). There have been investigated 60 hypo(an)ergic, chronic patients, with different causes of PICS including: chronic sepsis; massive polytraumatism; pancreatic necrosis; postoperative artificial intestinal fistulae; maldigestion and malabsorption syndrome; an immunologically compromised condition. There have been examined L.F. Gentille's determinants, which have confirmed the presence of PICS and patients have been administred an extracorporeal bio-xeno perfusion (timomyelo-spleen perfusion). To all of these patients the bioperfusion has been administered in accordance with the standard treatment: antibacterial and immunotropic therapy, surgical correction, mechanical methods used in MOST within the complex of extracorporeal detoxification, which proved to be resistant to treatment and financially costly. There were investigated A): L.F. Gentille's determinants; 1) BMI according A. Qetelet's formula (1869): BMI=m/h2( $\left.\mathrm{kg} / \mathrm{m}^{2}\right)$, where $\mathrm{m}=$ weight in kilograms; $\mathrm{h}=$ height in meters; 2) ICG according to correspondence of creatinine increase in urine (ICG=amount of creatinine measured in urine/creatinine parameters of a person having a corresponding height x 100); B) other tests: 1) the endogenous intoxication level "medium and low mass molecules" (MMM) after N.I. Gabrielean modified by M.I. Malakhova; 2) The number of bacterial cells was calculated through the Perfiliev capillary using N.S. Egorova's formula (1983): NCB=NMCBx10²/QLDxh, where NCB stands for the number of bacterial cells, NMCB represents the average number of bacterial cells in the Perfiliev capillary having the D length within the visual field; Q - capillary height, mm; L - the width of the capillary, mm; D - the diameter of the capillary within the visual field, $\mathrm{mm}, \mathrm{h}$ - dilution of the test material, $10^{3} \mathrm{x}$ conversion factor $\mathrm{cm}^{3} \mathrm{~mm}^{3} .3$ ) The skin bactericidal activity was evaluated according to N.N. Klemparski. The principle of the method: On the skin of the forearm, a culture of E. coli is applied. At regular intervals the number of viable bacteria is determined via the footprint method. Based on these results the bactericidal index is calculated (bactericidal index - BI) according to the formula: $\mathrm{BI}=\mathrm{CO}-\mathrm{C} 20 / \mathrm{COx} 100$, where $\mathrm{CO}$ stands for the number of colonies present immediately after skin contamination; C20 - the number of colonies observed after 20 minutes. Normally, BI constitutes $90-100 \%$. 4) Evaluation of complete phagocytosis. The principle of the method: E. coli is introduced into the citrate blood and is inoculated in a culture medium through a dimensional inoculation. After 120 minutes of incubation within the thermostat, a dimensional inoculation is repeated and both inoculations are incubated in a thermostat. In a day colonies are counted and the percentage of completion of phagocytosis (phagocytosis completion - FC) is calculated according to the formula: $\mathrm{FC}=\mathrm{CO}-\mathrm{CO} 120 / \mathrm{COx} 100$, where, $\mathrm{CO}$ represents the number of colonies immediately after inoculation; CO120 = the number of colonies after 120 minutes. Normally, complete phagocytosis constitutes-50-99\%. 5) The complete phagocytosis index (Complete phagocytosis index - CFI). Normally, after 30 minutes the neutrophils start digesting the microorganisms and after 2 hours it should end. Therefore, the percentage of phagocytic neutrophils and the average number of phagocytized microorganisms after exposure for 2 hours is smaller compared to the one recorded after 30 minutes, and CFI = average number of phagocytized microorganisms after $30 \mathrm{~min} /$ average number of phagocytized microorganisms After 120 minutes) bigger than 1.0 indicate a complete phagocytosis, and if the index is less than 1.0, this indicates a reduced function of killer neutrophils.

\section{Results}

\begin{tabular}{|l|c|c|c|}
\hline \multicolumn{1}{|c|}{ Marker/index } & Pre-bio-perfusion & $\begin{array}{c}\text { After bio-perfusion combined } \\
\text { the first day }\end{array}$ & $\begin{array}{c}\text { After bio-perfusion combined the } \\
\text { second day }\end{array}$ \\
\hline $\begin{array}{l}\text { Medium and low mass mol- } \\
\text { ecules (MMMem) erythrocyte } \\
\text { membranes, conventional units. }\end{array}$ & $0,508 \pm 0,06$ & $0,196 \pm 0,09$ & $0,127 \pm 0,08$ \\
\hline $\begin{array}{l}\text { MMMp plasma, convention- } \\
\text { alunits. }\end{array}$ & $0,678 \pm 0,09$ & $0,287 \pm 0,04$ & $0,237 \pm 0,03$ \\
\hline $\begin{array}{l}\text { MMMu in urine, conventional } \\
\text { units. }\end{array}$ & $0,110 \pm 0,09$ & $0,390 \pm 0,2$ & $0,420 \pm 0,1$ \\
\hline Bacterial cells, absolute & $2862 \pm 124$ & $106 \pm 12$ & $78 \pm 8$ \\
\hline C-reactive protein C, mkg/dl [7] & $210 \pm 14$ & $130 \pm 11$ & $90 \pm 08$ \\
\hline $\begin{array}{l}\text { The total number of lympho- } \\
\text { cytes }\end{array}$ & $560 \pm 23$ & $850 \pm 34$ & $1100 \pm 52$ \\
\hline Albumin, g/dl & $1,5 \pm 0,07$ & $1,7 \pm 0,08$ & $2,2 \pm 0,09$ \\
\hline Pre-albumin, mkg/dl & $7 \pm 0,1$ & $12 \pm 0,2$ & $16 \pm 0,3$ \\
\hline Appetite & absent & insignificant & good \\
\hline Body temperature in ${ }^{\circ} \mathrm{C}$ & $35,3 \pm 0,1$ & $36,8 \pm 0,2$ & $36,9 \pm 0,1$ \\
\hline Skin bactericidal Index, $\%$ & $26 \pm 3$ & $80 \pm 0$ & $95 \pm 2$ \\
\hline Complete phagocytosis, $\%$ & $7 \pm 1$ & $58 \pm 3$ & $90 \pm 2$ \\
\hline Complete phagocytosis index & $<1,0$ & $=1,0$ & $>1,0$ \\
\hline
\end{tabular}

Table 1: The antibacterial, anti-inflammatory, anti-immunosuppressing detoxifying and filtering functions observed after bio-xeno-myelo-timo-spleen perfusions $(\mathrm{M} \pm \mathrm{m}, \mathrm{n}=60)$ 
The analysis of this table shows a biosorbtion and filtering activity which occurred as a result of bio-perfusion and which is expressed in the desorption MMMem on the bio-membrane glycocalyx while the broken MMMem should have increased plasma levels of poisoning (MMMP) thus causing an artificial hipertoxoplasmy, but this has not happened, because the markers of endogenous intoxication in plasma are diminishing due to the effect of the cytokine "cascade" made, on one hand, by lysing the MMM oligopeptides (endogenous proteolysis of fermentomodulation), and on the other hand, the bioperfusion contributes to the improvement of the urine flow which increases the MMMu in the urine. The increase in the number of lymphocytes after the bio-perfusion corresponds to the law of lymphocyte activation and proliferation in direct not syngeneic contact with the cytokines of xeno-organs. The intense lymphoblastic transformation process after bio-perfusion activates immune suppression. The activation of cellular immunity optimizes local inflammatory response. This can also be seen from the increase in phagocytic activity of leukocytes confirmed by the activation of bactericide index of the skin. Further increase in the phagocytic activity together with the increasing lymphocyte mass leads to the stimulation of the humoral immunity. Not only the suction-filtration mechanism, but also the therapy action of anti-infectious cytokines leads to the diminishing of bacterial colonies relieving the body of pharmaco-chemotherapy complications whose massive antibacterial and fungicidal lysis effect can cause therapeutic endotoxemic shock similar to the Jarisch - Herxheimer syndrome or a disbiosis that will last over a longer period. The decrease in C-reactive protein as a result of bio-perfusion administration indicates a reduction of the anti-inflammatory activity, catabolism by promoting anabolism. Appetite restoration after bio-perfusion in the case of patients suffering from CARS, PICS and MODS made possible the adequate enteral nutrition by maintaining the respiration coefficient within an emergency unit. There have been used the recommendations of the American Association of Thoracic Surgery using "immune stimulatory" mixtures containing arginine, glutamine, nucleotides and/or omega-3 fatty acids (fish oil). As a result, after the 10th day post bio-perfusion, there has been attested a reduction of pain syndrome and hypothermia, patients' "creatinine growth" index, serum albumins, and IMC began to normalize. The absence of hypoalbuminemia manifestations, and even attempts to increase them after bio-perfusion are explained by the likelihood of extravasation of albumin and anabolic activation by counter-catabolic cytokines eliminated by the xeno-organs responsible for the homeostatic metabolism balance. The mechanism of reducing the albumin extravasation after bio-perfusion is also important because it locks the capillary leak syndrome, as a mandatory syndrome of overall edemation observed in MODS manifestations [8].

\section{Conclusion}

1. The extracorporeal perfusion in CARS, PICS, MODS situations, provides simultaneously both the mechanical treatment (mechanical filter - reducing the markers of endogenous intoxication) and the biodepurification (biological filter) that helps reduce microbial cells and endogenous toxic substances in all areas of vital importance: plasmic, biomembranic and the cytoplasmic.

2. In case of CARS, PICS, MODS syndromes, the bio-myelo-timo-spleen extracorporeal perfusion acts not only as a mechanical and biological filter, but also as an active phagocyte xenogeneic transfusion, leading to an increase of the complete phagocytosis and skin bactericidal index equivalent to the favoring cellular immunity and the optimization of the local inflammatory response syndrome (LIRS), which annihilates the exacerbation of generalized-CARS aggressiveness thus substituting the reduced macrophage phagocytic function and reversing the immune paralysis.

3. After the bio-myelo-timo-spleen extracorporeal perfusion in the context of CARS PICS and MODS syndromes, there is observed a regression of the immune paralysis (excessive anti-inflammatory response) due to the elimination of cytokines and leikines from the blood flow of the recipient by means of extracorporeal donor xeno-phagocite transfusion that balances the discordance of several pro and anti-inflammatory mechanisms and establishes the immune homeostasis. In this case, this method is considered to be a new-xeno-citokino (leikino) transfusion.

4. The Bio-myelo-timo-spleen perfusion compensates the extremely abnormal myelopoiesis in the post bio-perfusion because the appetite is restored, the body mass index is increased, "the creatinine growth" index is normalized, anemia is suppressed, pain and hypothermia is reduced, which indicates a regression of catabolism and an activation of anabolism.

5. The reduction of inflammation after bio-xeno perfusion directly points to the reduction of immune suppression and to the stabilization of disturbed processes between the humoral and the cellular immunity, which need the energy that is formed anew from pre-infusion hypo(an)ergic mitochondria.

6. The bio-myelo-timo-spleen perfusion supports the basic metabolism by activating anabolism and by suppressing catabolism, the syndrome of persistent inflammation and immune suppression, thus acting as an energo-modulator of hypo(an)ergic mitochondria whose lysosomal clearance (mitophagy) is affected as a result of cell death or apoptosis.

7. Thus, the bio-myelo-timo-spleen extracorporeal perfusion presents itself not only as a mechanical filter and biological activator of phagocytosis, but also as a stimulated energo-modulator of xenocitokino (leikino) transfusion within the CARS, PICS and MODS syndromes, which lacks a mechanical MOST.

\section{References}

1. Bone RC (1996) Toward a theory regarding the pathogenesis of the systemic inflammatory response syndrome: what we do and do not know about cytokine regulation. Crit Care Med 24: 163-72. 
2. Bone RC (1996) Immunologic dissonance: a continuing evolution in our understanding of the systemic inflammatory response syndrome (SIRS) and the multiple organ dysfunction syndrome (MODS). Ann Intern Med 125: 680-7.

3. Fumeaux T, Pugin J (2002) Role of interleukin-10 in the intracellular sequestration of human leukocyte antigen-DR in monocytes during septic shock. Am J Respir Crit Care Med 166: 1475-82.

4. Boomer JS, To K, Chang KC, Takasu O, Osborne DF, et al. (2011) Immunosuppression in patients who die of sepsis and multiple organ failure. JAMA 306: 2594-605.

5. Vanzant EL, Lopez CM, Ozrazgat-Baslanti T, Ungaro R, Davis R, et al. (2014) Persistent inflammation, immunosuppression, and catabolism syndrome after severe blunt trauma. J Trauma Acute Care Surg 76: 21-9.

6. Gentile LF, Cuenca AG, Efron PA, Ang D, Bihorac A, et al. (2012) Persistent inflammation and immunosuppression: a common syndrome and new horizon for surgical intensive care. J Trauma Acute Care 72: 1491- 501.

7. Flanders SA, Stein J, Shochat G, Sellers K, Holland M, et al. (2004) Performance of a bedside C-reactive protein test in the diagnosis of community-acquired pneumonia in adults with acute cough. Am J Med 116: 529-35.

8. Simbirtsev AS (2002) Cytokines - a new system of regulation of defense reactions. Cytokines and Inflammation 1: 9-16.

9. Vasiliev I, Creciun A (1986) Cazurile şocului anafilactogen şi tactica terapiei de urgenţă. Ocrotirea sănătăţii 5: 51-3.

10. Vasiliev I, Ceban N (1989) Terapia limfotropă cu limfostimulare în tratamentul complex al şocului exotoxic. Ocrotirea sănătăţii 2: 53-4.

11. Vasiliev I, Solodkin S, Nistor V (1993) Bioxenoperfuzia ca component terapeutic complex al tratamentului septicemiei pe fondul traumei asociate în condiţiile spitalului raional central. Curier medical. 2: 56.

12. Vasiliev I, Malachi M, Niculita I (1996) Bioxenosplenotimoperfuzia pentru tratamentul complex al lupusului eritematos sistemic. Curier medical 2: 41-3.

13. Vasiliev I (2009) Respiratory support with alveolar recruitment for complex treatment of acute lung injury and acute respiratory distress syndrome. Book of Abtracts III International Congress for Respiratory support Krasnoyarsk 25-7.

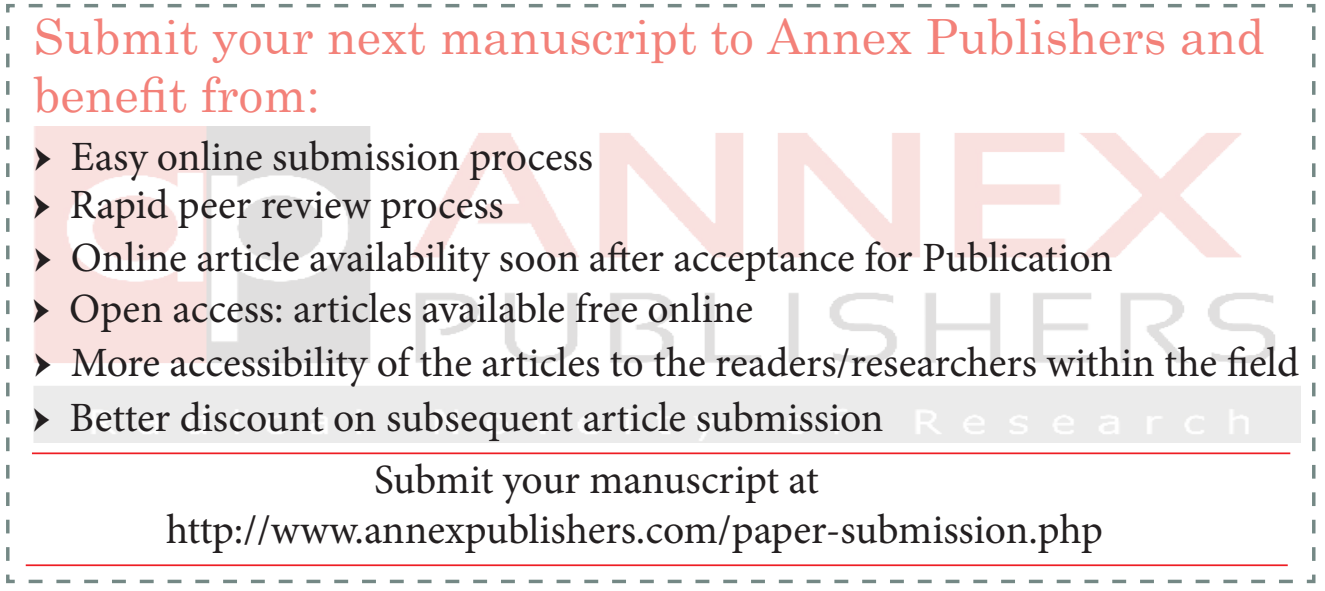

